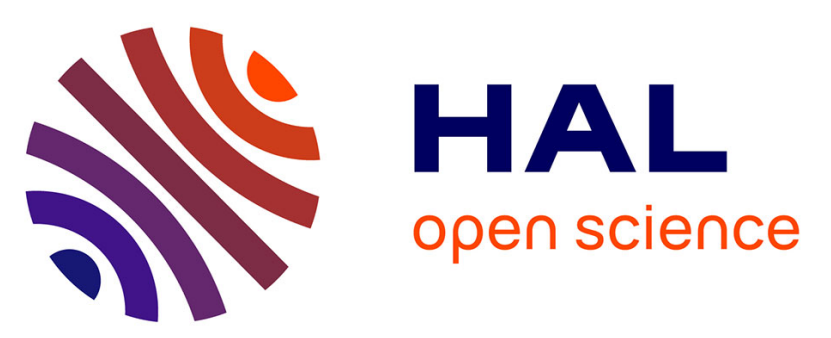

\title{
L'ambivalence du sujet au coeur de la flexibilité de la formation et de l'emploi : le cas des auditeurs du Cnam-Pays de la Loire inscrits en formation ouverte et à distance
}

André Moisan

\section{To cite this version:}

André Moisan. L'ambivalence du sujet au coeur de la flexibilité de la formation et de l'emploi : le cas des auditeurs du Cnam-Pays de la Loire inscrits en formation ouverte et à distance. Distances et savoirs, 2007, Industrialisation, repérages, vol.5 ( $\left.\mathrm{n}^{\circ} 1\right)$, pp. 83-117. 10.3166/ds.5.83-117 . edutice00183303

\section{HAL Id: edutice-00183303}

https://edutice.archives-ouvertes.fr/edutice-00183303

Submitted on 6 Mar 2008

HAL is a multi-disciplinary open access archive for the deposit and dissemination of scientific research documents, whether they are published or not. The documents may come from teaching and research institutions in France or abroad, or from public or private research centers.
L'archive ouverte pluridisciplinaire HAL, est destinée au dépôt et à la diffusion de documents scientifiques de niveau recherche, publiés ou non, émanant des établissements d'enseignement et de recherche français ou étrangers, des laboratoires publics ou privés. 


\title{
L'ambivalence du sujet au cœur de la flexibilité de la formation et de l'emploi
}

\section{Le cas des auditeurs du Cnam-Pays de la Loire inscrits en formation ouverte et à distance}

\author{
André Moisan \\ Lise (Laboratoire Interdisciplinaire de Sociologie Économique) \\ Cnam, UMR CNRS $n^{\circ} 6209$ \\ Case 250, 2, rue Conté \\ F-75003 - Paris \\ andre.moisan@cnam.fr
}

\begin{abstract}
RÉSUMÉ. Depuis près de 20 ans, le Cnam-Pays de la Loire développe une offre de formation à distance: près de 20000 auditeurs s'y sont inscrits. Une enquête téléphonique auprès de 352 d'entre eux, prenant le relais de 25 entretiens approfondis, met à jour l'adéquation de cette offre flexible avec la flexibilité du marché du travail et de l'emploi. Elle tend, par ailleurs, à faire émerger un individu ambivalent, privilégiant l'opportunité de s'affranchir de contraintes spatiales et temporelles sur la dimension pédagogique, appréciant l'autoorganisation par la FOAD tout en éprouvant son coût, à la recherche d'une reconnaissance de soi à la hauteur de l'engagement dans son parcours de formation. Elle atteste d'un déplacement de l'institution formatrice, d'une fonction socialisatrice à une fonction d'accompagnement.

ABSTRACT. For the last twenty years, the Cnam-Pays de la Loire has been developing a distance learning offer: around 20000 learners have been registered. A telephone survey addressed to 352 among them, after 25 in-depth interviews, revealed the adequacy of this flexible offer with the flexibility of the work market and employment. It also showed the emergence of an ambivalent individual who prefers to disenfranchise himself from the space and time constraints over the pedagogic dimension, preferring self-management, enabled by $O D L$, while bearing the brunt of its cost; seeking self-recognition as high as the level of his commitment to his training. Thus the institution that provides the training has shifted from a function of socializing to that of coaching.
\end{abstract}

MOTS-CLÉS: formation à distance, formation ouverte, formation ouverte et à distance, autoformation, flexibilité, société du risque, reconnaissance, relation emploi-formation.

KEYWORDS: distance learning, open learning, distance and open learning, self-directed learning, flexibility, a risk society, recognition, relation employment-training.

DOI:10.3166/D\&S.5.83-117@Cned/Lavoisier

D\&S - 5/2007. Industrialisation, repérages, pages 83 à 117 


\section{Introduction}

Des politiques actives sont mises en œuvre pour le développement des Nouvelles Technologies de l'Information et de la Communication dans le domaine de l'enseignement supérieur (TICE). En 2001, le ministère français de l'Education lançait son deuxième appel d'offres «Campus numériques» pour inciter les universités au développement de formation en lignes. Phénomène national, mais aussi international: J. Perriault, après avoir recensé les mesures d'incitation et de développement entreprises sur les deux rives de l'Atlantique, n'a pas peur d'énoncer «(...) qu'Internet, qu'on ignorait dans ce domaine jusqu'en 1997, se situe désormais - sans période probatoire - au cœur des politiques et des stratégies en matière d'éducation et de formation» (Perriault J., 2002, p. 231).

Ces politiques veulent prendre leur cohérence dans la visée globale d'une «société de l'information » : le sommet européen de Lisbonne de 2000 a ainsi défini une stratégie orientée vers cet horizon de développement, en mettant l'accent sur la formation tout au long de la vie pour favoriser la compétitivité et le développement. Ces stratégies trouvent leur déclinaison au niveau national et régional, s'articulant parfois avec une dimension de développement territorial ${ }^{1}$. De façon plus modeste, mais aussi plus ancienne, des acteurs régionaux ont perçu le défi (et l'atout) que pouvaient représenter les outils de communication à distance dans le développement économique local. C'est ainsi que des responsables politiques de la Région Pays de la Loire prenaient la mesure d'une politique active pour le maintien et le développement d'une offre de formation professionnelle supérieure au plus près des bassins d'emploi de la région, condition pour éviter la «fuite des cerveaux » de personnels qualifiés vers les métropoles régionales ou nationales. En 1986, le Cnam des Pays de la Loire était ainsi sollicité et subventionné pour mettre en place une offre à distance, en utilisant les outils de communication de l'époque.

Quelles que soient les vicissitudes que connaissent ces politiques et dispositifs, que sait-on des effets qu'ils produisent? Dans la production scientifique, comme dans celle produite par les acteurs, les analyses portent le plus souvent soit sur les usages et pratiques, en se restreignant à celles qui se développent à l'intérieur du dispositif et en privilégiant souvent la dimension pédagogique ${ }^{2}$, soit sur une analyse de type macro-économique et macro-sociale de l'industrialisation de la formation (Moglin P., 1998).

Entre les deux approches, qu'en est-il des effets de l'offre de formation supérieure professionnelle à distance quant à la relation formation-emploi ? Plus précisément, est-il possible d'analyser des transformations dans la façon dont des

1. Ainsi, les points d'accès régionaux à la formation, l'appel d'offre «FORE» pour le développement de ressources éducatives, (Ministère de l'Emploi et de la Solidarité, Programme national d'action pour l'emploi 2001).

2. Les travaux d'E. Kessous et J.-L. Metzger (cf. Kessous E. et al., 2005) et Fusulier B. et Lannoy P. (Fusulier B. et al., 1999) font exception. 
individus gèrent leur propre parcours de formation et leur parcours professionnel, du fait du développement de l'offre à distance ?

Une telle observation nécessite une certaine durée du dispositif : le Cnam-Pays de la Loire le permettait, puisqu'une politique constante de mise en œuvre s'ancre dans le territoire régional depuis presque 20 ans. Cette observation pouvait donc être conduite auprès d'une population significative et diversifiée, quant à ses caractéristiques sociodémographiques, ses projets formatifs et professionnels. De plus, l'unité spatiale était circonscrite : la région. Enfin, les caractéristiques de l'offre du Cnam régional - les mêmes formations sont proposées dans les deux modalités, à distance et en présentiel - permettaient d'établir des comparaisons entre deux populations, suivant les modalités de formation suivies.

Ces raisons ont plaidé pour la recherche dont il va être question $\mathrm{ici}^{3}$. Après avoir pris connaissance de l'offre de formation du Cnam Pays de la Loire, et du développement de la FOAD (Formation ouverte et à distance) et défini la méthodologie de l'enquête, elle s'est attachée à mettre en relation les caractéristiques de cette nouvelle offre avec l'évolution du marché du travail et de l'emploi, caractérisé par la flexibilité, pour en dégager les transformations qu'induit l'usage de ses ressources formatives chez les individus et leur rapport à l'environnement.

\section{Une offre de formation saisie à partir des parcours de formation à l'échelle régionale}

L'origine de l'offre de formation du Cnam-Pays de la Loire est importante à situer. Elle a été énoncée en introduction : elle est le produit d'une visite d'études des responsables politiques de la Région au Québec, au milieu dans années 1980. Ces derniers prennent la mesure de l'enjeu stratégique de la formation et de l'offre à distance, pour maintenir la «matière grise » dans les bassins d'emploi de petite et moyenne importance qui caractérisent la région : seule une offre de proximité peut en effet répondre aux besoins de maintien et d'évolution des compétences de cadres et de techniciens et éviter une «fuite des cerveaux » vers les métropoles régionales et nationales. En 1986, le Cnam-Pays de la Loire est ainsi sollicité et subventionné pour mettre en place cette offre à distance et la rapprocher des bassins d'emploi, en utilisant les outils de communication de l'époque.

En 1987 le Cnam-Pays de la Loire propose donc ses "téléformations" pour offrir, sous une forme suffisamment souple, à toute personne éloignée des grands centres urbains (où fonctionnent en général les Centres du Cnam) un accès à des cursus qualifiants et diplômants.

3. Cette recherche a été financée par le Ministère de la Jeunesse, de l'Éducation et de la Recherche. Elle a fait l'objet d'un rapport: Moisan A. (sous la direction de), Moreau J., Meyer A., Usage des dispositifs FOAD utilisant les TICE et leurs effets sur les trajectoires de formation et d'emploi, Décembre 2005. 
Depuis les 47 premières inscriptions de «télé-apprenants » en avril 1987, plus de 20000 personnes ont bénéficié des téléformations du Cnam-Pays de la Loire, et le « modèle » mis en place s'est déployé progressivement sur l'ensemble du réseau des centres régionaux du Cnam et du Cnam de Paris.

\subsection{D’une distance conjuguée à un ancrage territorial de proximité... à une distance intégrant l'individu dans un dispositif virtuel}

Les responsables du Cnam-Pays de la Loire répondent à la demande de la Région en mettant en place des « antennes », en complément des « centres d'enseignement». Les auditeurs s'y retrouvent au moins un soir par semaine: ils y trouvent l'équipement informatique et les outils de communication à distance qu'ils n'ont pas à domicile, le groupe et les pairs, mais aussi un responsable de l'antenne pour les aider tant d'un point de vue technologique que méthodologique, ainsi qu'une possibilité de communication (par téléphone et/ou minitel et/ou transpac) avec le formateur présent au centre de Nantes, sur des plages horaires convenues.

En 1997 (année d'activité maximum des antennes), le Cnam-Pays de la Loire dispose de 18 antennes multimédias réparties sur la région :

- 7 salles implantées dans les villes disposant d'un Centre d'enseignement «présentiel » (pour des enseignements non proposés en présentiel par ces centres...),

- 11 salles implantées dans les bassins d'emplois de 10 à 20000 habitants.

Ces 11 antennes sont mises en place en collaboration avec les communes ou les communautés de communes concernées.

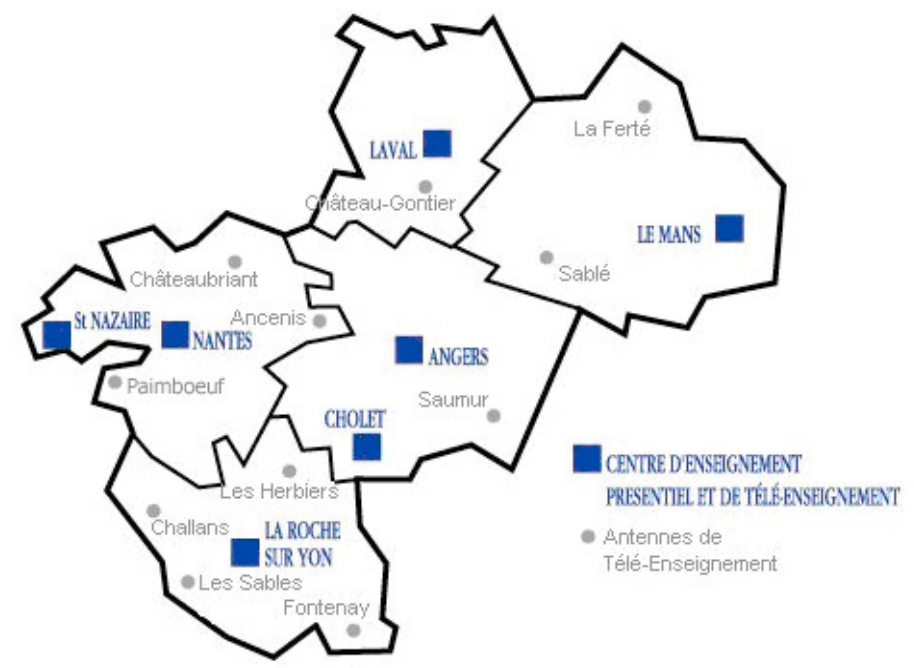

Implantation des centres et des antennes en 1998 
Autour des années 2000, au fur et à mesure de l'équipement personnel des auditeurs en micro-ordinateur et des développements Internet, ces antennes vont être désertées par les auditeurs. Cette désertion va, d'ailleurs, avoir des effets pervers : le taux d'abandon semble s'être renforcé dans cette période, l'isolement des auditeurs n'étant pas compensé par l'offre d'accompagnement via Internet qui va se mettre en place au fur et à mesure du développement des nouveaux outils de communication : essentiellement les messageries et les forums.

C'est la plate-forme Plei@d (pour Pays de la Loire Enseignement Individualisé à Distance) qui va prendre le relais en offrant cet environnement: conçue par le Cnam-Pays de la Loire à partir de 1997 et retenue en 2000 comme plate-forme nationale par le Cnam au niveau national, elle met à disposition les interfaces et toutes les fonctionnalités de la formation à distance.

Les auditeurs vont donc pratiquer leur formation essentiellement à domicile, parfois dans leur contexte professionnel. Ils ont également toujours accès aux salles implantées dans les centres d'enseignement présentiel : lors du démarrage de leurs formations les apprenants peuvent ainsi trouver dans ces antennes une initiation et un accompagnement technologique pour l'utilisation des outils proposés.

Mais, pour l'essentiel, la relation au dispositif d'offre de formation est passée d'un contexte de groupe de proximité locale à une relation individuelle reliée aux ressources via les outils de communication Internet.

\subsubsection{Une formation à distance intégrée à une offre singulière}

Mais avant tout, le Cnam est une institution d'enseignement supérieur professionnel qui s'adresse essentiellement à des adultes en activité ou en recherche d'emploi. De ce fait, l'offre à distance vient s'intégrer dans des filières, diplômes et des modalités singulières.

\section{Une offre modulaire}

L'offre de formation du Cnam se décompose en modules, appelées Unités de Valeur ou UV (depuis la réforme LMD, elles sont appelées : unités d'enseignement, soit UE). Chaque Unité de Valeur, d'une durée de présence élève variant de 80 à 120 heures (dans le nouveau dispositif, une UE correspond à 4 ou 6 ECTS), peutêtre suivie au cours d'une année universitaire. Il exige concrètement, en présentiel, une disponibilité d'au moins une soirée sur l'ensemble de l'année (ou d'un semestre, pour une UE).

Chaque UV (ou UE) fait l'objet d'une validation spécifique et vaut en elle-même. Elle peut s'ajouter à d'autres UV (ou UE) pour l'obtention d'un diplôme ou d'une 
certification $^{4}$ (l'équivalent d'une année universitaire requérant environ 3,5 UV et 7 UE dans le nouveau dispositif).

Cette modularité a deux conséquences importantes : l'engagement en formation ne correspond pas nécessairement à l'obtention d'un diplôme. Des études, conduites par l'Observatoire des Études et Carrières du Cnam, ont montré que des auditeurs peuvent s'inscrire pour un besoin ponctuel de formation correspondant à une seule UV (ce qui est corroboré par notre enquête). Par ailleurs, les auditeurs qui veulent s'inscrire dans une logique diplômante ont le choix du nombre d'UV dans l'année universitaire et, donc, de la rapidité de leur parcours de formation.

\section{Une offre combinant plusieurs ressources à distance}

L'offre à distance du Cnam Pays-de-Loire a toujours été mise en œuvre avec un accompagnement de ressources, dont les moyens techniques ont évolué.

La première d'entre elles correspond au formateur ou tuteur, que ce soit dans le dispositif des antennes (les auditeurs pouvaient le joindre par téléphone et/ou par Minitel) ou dans le dispositif Plei@d (par messagerie, forum et chats). Mais il faut ajouter également que des séances en présentiel sont la règle, selon un rythme «type » d'au moins 3 séances : au début, à mi-parcours, et à la fin ${ }^{5}$.

La deuxième est constituée, évidemment, par les «pairs » : les auditeurs inscrits. De la co-présence physique (à l'époque des antennes) à l'échange par les moyens virtuels de communication (via forum et messagerie), ses modalités ont également évolué.

La mise à disposition de supports est passée progressivement de l'envoi de documents (ou polycopiés) par envoi postal à leur numérisation et leur disponibilité sur la plate-forme Plei@d. Les possibilités numériques enrichissent progressivement la diversité des supports : vidéos, simulations, etc. viennent compléter les fichiers «html » ou «pdf ». La scénarisation pédagogique a aussi tendance à se diversifier avec l'expérience des concepteurs.

\section{Une offre à distance qui s'est développée progressivement}

L'offre à distance a évolué selon ses modalités (nous l'avons vu plus haut, en retraçant l'histoire des antennes jusqu'à la mise en œuvre de Plei@d). Mais elle évolue sans cesse dans la mise en ligne des différentes Unités de Valeur. La transcription de ces dernières est en effet progressive : elle est passée de 4 modules, en 1987 à 71 UV à la rentrée universitaire de 2004. Ils couvrent tout le spectre disciplinaire proposé par le Cnam.

4. Des certificats professionnels et des certificats de compétences agrègent la validation de plusieurs UV (ou UE) dans une logique qui ne correspond pas à une logique de niveau.

5. L'introduction des studios de cours, depuis 2004, fait encore évoluer les modalités de contact et d'échange entre tuteurs et auditeurs. 
Les auditeurs ont donc été mis dans une situation d'avoir à combiner des UV en présentiel et des UV à distance, les capacités de choix entre les deux modalités s'ouvrant au fur et à mesure de l'enrichissement de l'offre.

Une offre... répondant à une demande significative

L'offre s'est donc développée progressivement, au rythme des développements techniques de communication, de leur mise en forme technologique (Plei@ $\mathrm{d}$ en particulier), mais aussi de la scénarisation des différentes UV. Encore faut-il que cette offre rencontre une demande...: l'histoire des TICE (Technologies d'Information et de Communication pour l'Enseignement) n'est pas avare de telles offres qui ont généré plus de discours que d'ancrage dans la réalité.

Les statistiques d'inscription par le Centre Régional Cnam-Pays de la Loire font pourtant apparaître une réalité de l'usage de cette offre : marginale à ses débuts (3\% des auditeurs s'inscrivant à au moins une Unité de Valeur à distance), elle s'intègre comme pratique courante chez les auditeurs : plus de la moitié d'entre eux suivent au moins une UV à distance à la rentrée 2004.

\subsection{Une enquête à partir des parcours de formation}

Pour saisir, et d'une certaine façon mettre à distance la formation à distance, la recherche en question se définit d'abord comme une étude de population, et plus précisément des «trajectoires » d'emploi-formation (d'où son titre) générées par la FOAD.

\subsubsection{Objectifs de la recherche}

Elle se donnait initialement 3 objectifs :

1) Caractériser sociologiquement les populations inscrites dans les deux modalités de formation (FOAD et traditionnel), et tout particulièrement celle qui a développé un parcours de formation significatif en articulant plusieurs unités d'enseignement, dont certaines en FOAD. Quels sont les mobiles qui présidaient à leur entrée en formation, et plus spécifiquement dans leur choix de la FOAD ? De quel type de capital $^{6}$ » disposaient les candidats à leur entrée en formation (culturel, social, économique, etc.) ? Quelle position avaient-ils dans la carrière professionnelle (entrée dans la vie active, mobilité en cours de carrière, etc.) ? Quel est l'effet de leur situation matrimoniale ? De l'éloignement du centre de formation ?

2) Identifier les pratiques et les usages des dispositifs de formation et de l'ensemble des ressources technologiques, pédagogiques et humaines qu'ils intègrent en distinguant les ressources formelles proposées à l'apprenant par le dispositif institué de celles qu'il a identifiées et utilisées dans sa sphère personnelle et/ou

6. Dans le sens de P. Bourdieu. 
professionnelle; d'identifier et d'évaluer les «effets de formation et d'apprentissage » induits plus spécifiquement par la FOAD (développement de capacités d'autodirection, construction de son propre environnement d'apprentissage, identification et mobilisation des ressources, évolution du rapport au savoir et aux institutions de formation, etc.)

3) Qualifier les mobilités de formation et les mobilités professionnelles à l'issue de ces formations.

\subsubsection{Ses principales hypothèses}

La méthodologie de la recherche se voulait inductive. C'est la raison pour laquelle il a d'abord été envisagé une enquête qualitative pour faire ressortir des hypothèses fortes sur la différentiation des populations. Il fallait remonter au projet de formation et au contexte de son élaboration (professionnel, en particulier) pour bien saisir les raisons du choix ou de la distance : l'hypothèse générale, bien que non écrite, était de faire prévaloir des « ressources stratégiques » plus fortes chez les auditeurs s'inscrivant en FOAD, dans leur capacités à s'affranchir plus facilement de la présence institutionnelle de la formation traditionnelle, de s'informer des modalités de formation non traditionnelles existant sur leur territoire et de se mobiliser en prenant appui sur un réseau d'aide de proximité pour conduire leur parcours de formation. C'est ce qui a orienté la constitution de la grille d'entretien pour la phase qualitative ${ }^{7}$.

Les travaux d'A. Jezegou ${ }^{8}$, par ailleurs, n'étaient pas sans nous indiquer que l'usage de la FOAD interrogeait tout particulièrement les stratégies «d'autodirection» des apprenants, celle-ci pouvant développer des apprentissages spécifiques dans la capacité à les mettre en œuvre et à les développer.

\subsubsection{L'enquête qualitative}

La première phase de l'étude a donc consisté à recueillir des matériaux d'analyse par des entretiens approfondis pour permettre de faire émerger un certain nombre de variables explicatives pouvant par la suite être testées par une étude quantitative.

25 entretiens ont ainsi été conduits, sur la base d'une sélection de 50 auditeurs dans le fichier de la scolarité, à partir des critères suivants :

7. L'adoption de cette méthodologie de type «longitudinale» correspond aux souhaits exprimés par des auteurs, critiques sur les types d'études majoritairement développés sur la FOAD (Bélisle C. et al., 2004) : «il semble que la finalité poursuivie dans les études soit la recherche d'un mode satisfaisant d'intégration des TICE dans l'exercice pédagogique", alors qu'il faudrait privilégier, dans ce «champ de pratiques» les notions de « configuration, de trajectoire, de parcours, de chemin. » ( p. 159)

8. Elle a participé à l'élaboration du projet de l'étude, et en particulier à la rédaction de la réponse à l'appel d'offres. Ses travaux portent sur le croisement de «l'ouverture des dispositifs » de formation et de « l'autodirection des apprenants ». 
1) type de cursus : inscription dans des filières et des cycles de formation où l'offre de la FOAD s'était développée de façon consistante («Economie-Gestion », Cycle A, «Informatique » Cycle A et Cycle B),

2) critère temporel : auditeurs ayant, pour 1/3 fini leur formation (au minimum 2 UV) depuis 2 ans ou plus, pour $1 / 3$ entre 1 an et 2 ans, et pour $1 / 3$ en cours de formation (inscrits de 2004-2005),

3) Critère spatial : Région Pays de Loire au moment de leur formation,

4) Critère sociodémographique : échantillon respectant en gros les proportions de la population des inscrits de ces filières en termes de genre, âge, situation matrimoniale, éloignement géographique du centre d'enseignement, répartition sur les 5 départements de la Région des Pays de Loire, situation professionnelle.

Les entretiens étaient de nature semi directive. Ils ont été conduits par les 3 chercheurs impliqués dans l'étude, à partir d'une grille d'entretien commune. La conduite de l'entretien devait aider l'interviewé à reconstruire et dérouler l'ensemble du processus de formation avec le Cnam, en partant de sa genèse et du contexte de son inscription. Il était ainsi initié par cette question : «Remettez-vous dans la situation où vous étiez au moment où vous avez fait le pas de vous inscrire au Cnam ». En aucun cas, dans l'annonce et l'ouverture de l'entretien, il n'était précisé que l'enquête portait spécifiquement sur la formation à distance. Il nous semblait, en effet, important de ne pas mobiliser cognitivement l'interviewé sur la modalité pédagogique, mais au contraire de voir comment elle émergeait dans la reconstruction de son parcours.

L'ensemble des entretiens a été retranscrit. Ils ont fait l'objet de nombreux échanges et confrontations entre les 3 chercheurs. Une grille d'analyse commune a été adoptée. Sont ainsi apparus des traits et caractéristiques qui ont été à la base de l'élaboration du questionnaire.

\subsubsection{L'enquête quantitative}

La seconde phase de l'étude a consisté à recueillir des matériaux d'analyse par des questionnaires approfondis. Ils devaient nous permettre de valider les hypothèses et variables explicatives qui avaient émergé dans l'enquête qualitative et notamment de comparer les profils et les pratiques des stagiaires inscrits à distance exclusivement, avec ceux qui suivaient les cours en présentiel exclusivement et ceux qui avaient mélangé les deux modalités.

352 questionnaires ont été recueillis parmi un échantillon d'inscrits au Cnam des Pays de la Loire avant 2003 (sources : fichier de la scolarité).

Les principaux critères retenus pour la constitution de l'échantillon étaient :

- le type de formation : 4 types ont été retenus, en référence aux pôles nationaux du Cnam : Informatique, Société, Technologie et Économie-Gestion, 
- le niveau de chaque formation Cnam suivie $(\mathrm{A} / \mathrm{B} / \mathrm{C}$, identifié à partir des derniers caractères de son intitulé),

- les Unités de Valeur pour lesquelles le choix entre la FOAD et le présentiel était possible,

- une inscription dans une période antérieure à 2003, de manière à ce qu'il y ait une probabilité plus grande d'avoir terminé leur formation.

Trois périodes ont en effet été distinguées en fonction des outils mis à disposition des stagiaires :

- la période des antennes (jusqu'en 1997), c'est la période où des auditeurs se retrouvent dans des lieux sans enseignants où ils travaillent en petit groupe sur des supports mis à disposition sur des ordinateurs non reliés,

- la période de transition (entre 1998 et 2000), c'est celle de la désertion progressive des antennes du fait de l'équipement des auditeurs en ordinateur, et de l'augmentation de l'accès à Internet ;

- la période PLEIAD (à partir de 2001), où une plate-forme Internet met en accès direct les ressources de formation sous le tutorat d'un enseignant. Cette plate-forme bénéficie des nouveaux outils des technologies de la communication (messagerie, chat...).

L'enquête a été réalisée par une équipe de 5 enquêteurs de la société G\&A. Les enquêteurs ont été formés avant le début de la passation et un débriefing a été organisé à son issue.

Une population cible de 2000 inscrits a reçu un courrier d'information les avertissant qu'ils allaient participer à une enquête sur les usages de la formation.

Les enquêtés ont été contactés par téléphone le soir entre 18 heures et 20 heures et le samedi matin. L'accueil a été très bon aux dires mêmes des enquêteurs. Le courrier envoyé en amont y a beaucoup participé. Les enquêteurs ont eu peu de réponses négatives (refus de répondre): 122, rapportés à 352 questionnaires recueillis au final. La durée moyenne de passation était de 20 minutes.

L'échantillon constitué est relativement fidèle à la population inscrite au Cnam traditionnellement. On notera toutefois l'existence de trois biais à prendre en compte pour l'analyse des résultats, qui ne les remettent pas pour autant en cause.

- l'information concernant la modalité que les enquêtés déclaraient était parfois assez différente de celle présente dans le fichier de la scolarité, notamment pour les plus anciens. Une partie des inscrits en FOAD se déclarent en fait «en cours du soir» (pour les plus anciens), et beaucoup se déclarent dans des parcours mixtes (pour les plus récents). Cette différence peut être liée au fichier lui-même (doublons, déménagements, etc.), à des formations récentes, ou à des oublis de la part des personnes interrogées. 
- plus de 600 courriers d'annonce ont été retournés à l'envoyeur, indice d'une forte mobilité des anciens stagiaires, qui par voie de conséquence, ont été exclus de l'enquête,

- la surreprésentation des femmes (49\% dans l'enquête contre 30 à $35 \%$ en moyenne chaque année dans la région). L'échantillon est représentatif en termes d'âge, de CSP, d'objectif de formation, mais les femmes y sont surreprésentées (sans doute parce que plus présentes que les hommes au sein de leur lieu d'habitat aux heures où se déroulaient les appels).

Les analyses dont il va être question ci-dessous prennent appui sur l'analyse de contenu des entretiens et sur le traitement statistique des données quantitatives et sur leurs résultats, publiés dans l'étude remise au commanditaire : le Ministère de la Jeunesse, de l'Éducation et de la Recherche. Elles ne reprennent pas à la lettre les objectifs de la recherche, tels qu'ils ont été énoncés plus haut : elles font prévaloir une grille d'analyse qui met en relation ces résultats avec une problématique générale de l'évolution des formes sociétales de la relation formation-emploi (que nous caractérisons par «l'époque de la flexibilité ») et de l'individu social.

\section{Un dispositif de l'époque de la flexibilité}

Par flexibilité, nous entendons d'abord la remise en cause de la forme sociétale «taylorienne-fordienne » ${ }^{9}$. Les grands ensembles organisateurs spatio-temporels, construits sur un mode homogène à partir des années 1920 jusqu'aux années 1980 (avec leur plein développement pendant ce qu'on a qualifié de «trente glorieuses»), se sont en effet diffractés. Les horaires de travail et les dates de congés se diversifient. Les statuts d'emploi ne répondent plus à la norme du contrat à durée indéterminée, en même temps que le temps partiel se développe. Les espaces-temps qui régulaient le parcours de vie tendent à se décloisonner (il est question d'une formation «tout au long de la vie », et non plus seulement d'une formation continue, comme prolongement d'une formation initiale). Il en va de même des normes de vie familiales, sexuelles, etc. : le modèle de la famille biparentale, élevant deux enfants, fait de moins en moins référence. Les classes et strates sociales perdent leur caractère homogène unificateur.

Cette diversification relève autant de politiques mises en œuvre que de choix de modes de vie et de possibilités offertes par l'évolution technique (en premier lieu, les technologies de l'information et de la communication). Mais par «flexibilité », nous voulons inscrire les résultats de cette recherche à partir du cadre de pensée développé tant par les sciences sociales que par les acteurs de la vie économique concernant la relation formation-emploi, à partir des premiers travaux de l'OCDE du

9. Nous utilisons ici les grandes catégories des économistes de l'école de la régulation. 
milieu des années $1980^{10}$. Cette notion est mobilisée le plus souvent de deux points de vue contradictoires: soit pour afficher un volontarisme politique contre les « rigidités » du marché du travail, soit d'un point de vue critique, pour faire valoir la vulnérabilité sociale, conséquence de la précarité.

Nous situerons ici la «flexibilité » comme une donnée : une nouvelle donne de cette relation formation-emploi, dont l'offre de formation à distance ne serait que la traduction dans le domaine de la formation par la mise à disposition de modalités qui lui correspondent.

\subsection{Le primat de la modularité}

Le premier aspect à noter correspond au fait que la modalité pédagogique correspondant à la «distance » n'est pas celle qui est privilégiée dans les raisons évoquées par les auditeurs pour s'inscrire au Cnam. Dans l'enquête téléphonique, ils avaient ainsi à indiquer leurs préférences sur 3 caractéristiques qui singularisent l'offre du Cnam : $53 \%$ des interviewés mettent en avant la dimension modulaire de la formation, $28 \%$ son caractère professionnel et seulement $17 \%$ le choix de la modalité.

Un auditeur, dans les entretiens qualitatifs, le témoigne à sa façon. Il s'était inscrit à une formation à distance auprès d'une université, formation qu'il a arrêtée étant donné la rigidité de son organisation en années universitaires.

(...) je ne me souviens pas, (...), si on avait le choix du nombre d'UV dans l'année ou si on avait un temps imparti, alors qu'au Cnam, on peut s'inscrire à une UV et ça peut durer 3 ou 4 mois et puis on laisse et on repique l'année d'après... Parce que quand on travaille, et qu'on a une famille en plus... pouvoir assumer les deux, c'est long...

Ce résultat est confirmé a contrario par la réponse à une question sur le principal inconvénient de cette même offre. Si le coût de l'inscription est mentionné en premier par $16 \%$ des interviewés et la durée des études par $22 \%$ d'entre eux, ils sont plus nombreux à considérer que le principal inconvénient correspond au fait que «tous les cours ne soient pas ouverts tous les ans $»^{11}$.

Autrement dit, la modalité pédagogique (présentielle ou à distance) n'est perçue ici que comme un renforcement de la flexibilité introduite par la modularité de la formation.

10. Cf. en particulier les développements de R. Boyer (1986), économiste appartenant à cette école de la « régulation».

11. Certains centres d'enseignement ouvrent les UV une année sur deux, pour s'assurer d'effectifs suffisants. 


\subsection{Une préférence affichée pour le modèle pédagogique traditionnel}

Les auditeurs expriment massivement leur préférence pour les modalités pédagogiques des cours du soir : les auditeurs qui ont suivi des UV en FOAD, dans leur majorité, considèrent qu'ils auraient mieux appris en formation traditionnelle.

Ainsi, «avec le recul, une fois l'expérience faite de la formation à distance », ils sont $51 \%$ (des 142 répondants inscrits en FOAD) à porter un tel jugement (et $39 \%$ qu'ils auraient appris «de façon équivalente», $6 \%$ seulement estimant qu'ils auraient «moins bien appris »).

Et pour ce qui concerne les auditeurs ayant suivi un parcours mixte (UV en FOAD et en traditionnel), il ressort que $61 \%$ d'entre eux préfèrent les cours du soir et $68 \%$ qu'ils correspondent mieux à leur « façon d'apprendre».

De plus, quand on observe les arguments apportés par les 24 interviewés qui se déterminent pour la modalité FOAD comme correspondant à leur façon d'apprendre, seuls 7 d'entre eux sont strictement d'ordre pédagogique (les autres arguments relevant davantage des conditions dans lesquelles s'exercent l'acte d'apprentissage : choix de la plage horaire, gain de déplacements, etc.).

Cette préférence «pédagogique » au mode traditionnel ressort abondamment des entretiens qualitatifs. Ainsi, un auditeur (qui a suivi pendant plus de 10 ans des cours à distance, pendant la période des antennes) avoue :

Les cours magistraux, c'est nettement mieux. Quand on ne sait pas, on pose la question (...) quand on a quelqu'un de physique devant, il répond à la question. Tandis qu'avec la messagerie, c'est pas évident.

On suit bien cette controverse intérieure dans le choix de la modalité à travers les hésitations de l'auditeur suivant :

Je pense que j'aurais suivi les cours du soir parce qu'on est vraiment obligé d'être présent car à distance on télécharge les cours quand on a envie, on travaille quand on a envie c'est vrai que c'est beaucoup moins facile ...

... et il évolue :

$Q$ - (...) aujourd'hui, connaissant les 2 modalités, quel serait votre choix?

$R$ - Je pense que j'aurais pris la formation à distance quand même. C'est moins contraignant

... pour enfin avouer :

Je pense que si j'avais réellement le temps, j'irais en cours du soir.

Ces hésitations de l'auditeur sont emblématiques de la façon dont se construit le choix des auditeurs : le modèle pédagogique semble fonctionner comme «référence». Après tout, c'est le modèle scolaire qui façonne les représentations. Mais, entre cette vision idéalisée et le système de contraintes, l'auditeur va jouer de ces limitations imposées et des opportunités pour construire les modalités de son parcours de formation. 
Il est néanmoins possible de constater un effet d'apprentissage lié à la pratique de la FOAD. À la réponse à la question : «Après avoir suivi des enseignements à distance, avez-vous le sentiment d'avoir appris d'autres façons d'apprendre que celles de l'école ? », $78 \%$ de ceux qui ont utilisé la FOAD répondent positivement.

La réponse à une autre question donne des précisions quant à ces «autres façons d'apprendre » (liées, en l'occurrence ici, à la FOAD). À la question : «Avez-vous changé d'avis sur l'enseignement à distance après votre inscription », la majorité maintient sa représentation initiale (61\%). Mais ils sont près du tiers (29\%) à considérer qu'elle a évolué «plutôt en bien ». La préférence affichée pour le modèle pédagogique traditionnel semble donc surtout se vérifier au moment de la première inscription.

\subsection{L'avantage spatial et temporel de la FOAD}

Mais ce qui est notable, c'est que la dimension pédagogique n'apparaît pas comme premier dans la hiérarchie des préférences mobilisées par les auditeurs ayant fait le choix de la FOAD, soit comme unique mode de formation, soit combinée avec les autres modes de formation.

L'offre à distance se trouve en effet plébiscitée par les possibilités qu'elle donne d'échapper à des cadres spatio-temporels trop rigides. Une des questions portait explicitement sur les avantages de la FOAD, qu'elle demande de hiérarchiser dans une échelle de préférence. Elle a donné les résultats suivants :

\begin{tabular}{|l|c|c|}
\hline LA FOAD & $\begin{array}{c}\text { Les 2 items : } \\
\text { Tout à fait + Plutôt }\end{array}$ & $\begin{array}{c}\text { Item : } \\
\text { Plutôt (seul) }\end{array}$ \\
\hline évite des déplacements & $88 \%$ & $73 \%$ \\
\hline permet de travailler à sa propre vitesse & $87 \%$ & $68 \%$ \\
\hline $\begin{array}{l}\text { permet de continuer la formation en cas de } \\
\text { déménagement }\end{array}$ & $86 \%$ & $71 \%$ \\
\hline $\begin{array}{l}\text { permet de répondre aux contraintes } \\
\text { professionnelles }\end{array}$ & $85 \%$ & $64 \%$ \\
\hline permet d'organiser sa propre façon d'apprendre & $85 \%$ & $53 \%$ \\
\hline permet de répondre aux contraintes familiales & $68 \%$ & $53 \%$ \\
\hline offre un meilleur mode d'apprentissage & $30 \%$ & $12 \%$ \\
\hline
\end{tabular}

Liste des avantages de la FOAD, selon une échelle de préférence 
La faible propension à reconnaître des qualités pédagogiques à la FOAD se trouve confirmée ici par la faiblesse des réponses du dernier item (l'offre d'un meilleur mode d'apprentissage).

Mais les autres items font apparaitre en premier l'économie de déplacements, bien exprimée par l'extrait d'entretien ci-dessous :

Avec mon travail, ce n'était pas forcément facile parce que j'habite à $20 \mathrm{kms}$ du Mans, donc en fait pour venir au cours du soir, c'est de 18h à 21 heures, ou 20 heures, ça dépend des valeurs, c'est vrai que ça faisait lourd au niveau de la semaine, donc en fait dans la semaine je prenais moitié des valeurs sur place et moitié des valeurs à distance, par la plate-forme en fait. Et comme je me débrouillais assez bien toute seule (...).

Vient ensuite le critère «temporel», qui ne relève pas uniquement du libre choix de la plage horaire d'étude du cours. Les entretiens permettent d'affiner cette dimension.

Il est question (1) de rythme dans l'apprentissage :

Au niveau du rythme de travail, çà ne me correspondait pas [le présentiel], c'était des journées chargées. A ce moment-là, j'avais l'impression que j'avais des problèmes d'assimilation, que je n'ai pas retrouvés après... [dans les cours à distance].

(2) d'économie de temps par une organisation temporelle

L'avantage que j'ai vu par rapport au cours du soir, c'est qu'on peut choisir en fait quand est-ce qu'on prend les cours. On gagne vachement de temps, parce qu'on a pas besoin de noter les cours.

(3) mais aussi d'organisation des activités entre les temps dédiés à la formation et les temps d'activités de «temps libre»

$Q:$ Donc UV à distance, on vous propose les deux ou...

$R:$ Ah ben oui, je... surtout que même le soir où il y avait cours, je faisais du sport, je ne me voyais pas, j'aurais pu trouver d'autre soirées de libre, mais du coup, on n'en a plus vraiment de temps libre. Tous les soirs pris, non. Je trouvais que ça faisait beaucoup, ça me prend déjà 12 heures par semaines, donc.

Les auditeurs vont donc apprécier, jauger, arbitrer entre les préférences pédagogiques du traditionnel et les avantages de la distance. D'une certaine façon, contrainte et opportunité s'évaluent et s'éprouvent dans le même mouvement (nous y reviendrons au paragraphe 4.2). 


\subsection{Un usage différentié des ressources de formation}

Une autre caractéristique majeure des pratiques des auditeurs tient au fait de la diversification des choix et des usages. Elle se situe à trois niveaux.

3.4.1. Le choix de la combinaison entre les cours traditionnels (du soir) et les cours à distance

C'est ici que les auditeurs font pouvoir faire valoir leurs préférences, entre les aspects pédagogiques et les avantages spatio-temporels. L'expérience de certains auditeurs, par exemple, leur a appris que pour l'étude de disciplines fondamentales (mathématiques, économie générale), le présentiel est préférable. Ils vont donc combiner leur inscription en présentiel avec leur inscription en FOAD. Cet auditeur illustre assez bien, à la fois de la démarche en formation que de la façon dont s'opèrent les choix.

Je me suis dit étant donné que Clarté [centre lavallois de ressources technologiques] c'est une petite structure, on est peu nombreux, on est huit personnes, je ne vais pas pouvoir bénéficier de formation en interne et de quelqu'un qui pourrait m'aider sur un certain nombre de mois, donc je me suis dit «faut que je trouve une autre solution ». (...).

Ils [des enseignants] m'ont dit comment ça se passait, c'est le soir en général de $18 \mathrm{~h}$ à $21 \mathrm{~h}$. et puis il y a aussi des cours à distance avec un passeport et un ? On a accès à une plate-forme et on télécharge les cours de façon hebdomadaire ou bimensuelle, donc je me suis dit oui c'est intéressant, etc. Donc à la rentrée scolaire de septembre 2002, je me suis inscrit au Cnam et j'ai entrepris de faire un DEST, niveau BAC +4 , en système d'information, donc orienté réseau, système, etc. (...) C'était assez difficile parce que j'en avais deux en présentiel à Laval, plus les trois à distance à Nantes, bon ben je m'accrochais, je travaillais deux heures tous les soirs après le travail, puis je me servais aussi des vacances et mes journées RTT pour essayer de combler un peu le retard si j'avais pris du retard.

\subsubsection{Un usage différentié des ressources de formation}

Supports en ligne, mais aussi messagerie, forum, chat, séances en présentiel pour des relations entre pairs et tuteurs : c'est une diversité de ressources formatives qui est proposée en FOAD. Mais également : autres ressources consultables sur Internet, collègues de l'entreprise, amis, parents, association. Les 3 auditeurs, ci-dessous, en témoignent.

(1) $R$ - j’ai eu la chance que mon papa était dans le métier...(...) Je lui envoyais par Internet tous les cours, tous les exercices, les corrigés et dès que j'avais des questions, je lui envoyais des messages, et il regroupait, regardait quelle page ça se trouvait, voilà... 
(2) (...) tout en continuant à travailler, moi mes compétences elles augmentaient à vitesse grand $V$, parce que j'étais aussi en contact avec d'autres développeurs et d'autres informaticiens qui ne faisaient pas forcément $d u$ développement, ça fait un foisonnement de compétences réunies à un seul endroit, où on chope toutes les bonnes infos...

$Q$ - Alors qu'est-ce que ça vous apportait précisément les cours du Cnam? (...)

$R$ - Ca m'a consolidé mes connaissances, ça m'a déjà fortifié mes bases et puis ça m'a permis de voir des trucs que je ne voyais pas forcément au travail, donc ça a complété l'expérience professionnelle.

(3) Pendant que j'étais en cours, j'étais membre d'une association (...) Chaque fois que j'avais quelque chose dont j'avais besoin sur la programmation, etc., je me dirigeais vers mon association et puis j'avais les outils et l'environnement adaptés pour faire mes programmes. C'est grâce à ça entre autres que j'ai trouvé mon travail.

Ces pratiques ne sont pas réductibles au seul contexte de la FOAD (même si on peut penser que la situation d'isolement de l'apprenant peut le conduire à solliciter son entourage). Par contre, les auditeurs qui s'inscrivent dans les UV selon cette modalité ont le choix du type de relation avec le tuteur. Les outils de communication Internet, depuis Plei@d, semblent avoir développé ces relations (en comparaison avec la période des antennes).

Pour ceux qui développent cette relation avec le tuteur, il est possible de distinguer trois figures ${ }^{12}$ :

(1) La figure du précepteur: des auditeurs essaient de faire valoir une relation individuelle et privilégiée.

(2) La figure du « maître » professionnel, qui va transmettre l'habitus du métier :

$Q$ - Comment ça se passe concrètement ce contact humain?

$R$ - Ce contact humain c'est... comment vous dire une forme d'empathie, vous voyez ce que je veux dire, il y a une transmission intellectuelle mais en même temps il y a le comportement de la personne, son habitude, c'est tout ce jeu de tuteur qui fait qu'on aspire à quelque chose, à une connaissance qu'il peut nous apporter énormément.

(3) La figure de l'animateur collectif, qui initie, enrichit et veille à la qualité et au volume des échanges sur les forums et les chats.

12. D'autres fonctions peuvent également être repérées, à travers le besoin d'écoute (14\%), d'organisation méthodologique $(25 \%)$ et de motivation (17\%). Nous n'avons retenu ici que les figures qui émergeaient de façon dominante. Il faut préciser qu'elles correspondent aux figures attendues par les auditeurs, et non les pratiques observées des tuteurs. Cf. la typologie développée par V. Glikman (2002). 
Globalement, le croisement des interactions développées avec le tuteur et avec les pairs inscrits aux mêmes UV font apparaître: un noyau d'environ $1 / 3$ d'indépendants (se dispensant de toute interaction); une population d'auditeurs privilégiant une relation préceptorale (orientée uniquement vers le tuteur); et enfin une population de «socio-apprenants $»^{13}$ (s'emparant des outils de Plei@ $\mathrm{d}$ pour intensifier les échanges tant avec le tuteur qu'avec les pairs).

\subsection{Des effets limités d'accélérateur de carrière par les parcours « mixtes »}

Les auditeurs de notre enquête ont connu des formes de mobilité, voire des évolutions de carrière : $53 \%$ déclarent avoir changé de situation, de métier ou de statut par rapport à avant leur formation. Mais, outre qu'on ne peut pas imputer à la seule formation ces évolutions de carrière, on ne peut pas affirmer que la modalité de formation ait joué un rôle déterminant. Les $53 \%$ d'auditeurs en question se répartissent en effet de façon très homogène selon leur inscription en FOAD, en cours du soir ou en parcours mixte.

Les auditeurs inscrits dans les parcours mixtes sont pourtant légèrement plus nombreux à répondre qu'ils ont atteint les objectifs professionnels qu'ils s'assignaient au moment de leur inscription en formation.

Un autre effet identifiable, certes mineur, est celui de l'accélération des parcours de formation que permet la FOAD. On constate en effet que, dans une question qui porte sur le bilan de leur formation au Cnam, les auditeurs qui ont utilisé cette modalité sont plus nombreux à choisir l'item «m'a permis d'atteindre plus rapidement mes objectifs ». L'effet est encore plus marqué pour ceux qui ont opté pour un parcours mixte.

"Je pensais le décrocher plus vite, mais je pense que j’ai été plus vite avec l'enseignement à distance que si j'avais dû venir en cours. Je n'aurais pas tenu quatre ans (et ce n'est pas que de la validation d'acquis). Pour moi, c'est vraiment la bonne formule, et puis je ne sais pas si j'aurais pu suivre tout, parce que les cours tombaient les mêmes soirs ».

S'il est donc difficile de lire la traduction de cette offre flexible de formation dans la dynamique emploi-formation au niveau d'un territoire régional (qui supposerait un équipement statistique beaucoup plus ambitieux), il est néanmoins permis de voir comment sa flexibilité ouvre le champ des possibles pour des adultes (ou jeunes adultes) pour la plupart insérés dans la vie active. Elle propose des

13. Ce terme est repris de S. Alava (2002), qui définit 2 types d'usagers des supports de formation dans «l'enseignement ouvert»: les «autonomes» et les «socio-apprenants ». Le terme $d$ '«indépendant» nous semble mieux convenir: les «socio-apprenants » font également preuve d'autonomie. L'indépendance renvoie, ici, à l'absence d'interaction sociale avec les autres acteurs de l'intervention sociale. 
solutions pour échapper aux contraintes spatio-temporelles ; elle donne la possibilité à l'auditeur d'utiliser au mieux les ressources de formation (de l'offre et de son environnement) et ses propres caractéristiques d'apprentissage. La flexibilité du dispositif répond à la flexibilité des conditions de travail et de l'emploi et, ce faisant, elle participe à la construire et la développer.

Mais il est aussi une autre transformation que révèle la pratique par les auditeurs de cette offre : celle-ci se situe, non plus dans la dimension macrosociale de la relation formation-emploi, mais au niveau de l'individu lui-même.

\section{Un individu autodéterminé et ambivalent}

L'auditeur, avec la FOAD et sa combinaison avec le mode traditionnel de formation, voit donc s'ouvrir le champ des possibles. La «flexibilité » 1'y conduit (et l'y contraint) : il doit prendre l'initiative et assumer ses choix. Mais que peut-on dire de cet individu ? Se différentie-t-il des auditeurs du Cnam qui optent pour le mode traditionnel ? De façon plus précise, comment opère-t-il ses choix ? Nous avons pu apercevoir, dans les développements précédents et les extraits d'entretien, que l'initiative se mêlait à la contrainte: les auditeurs s'inscrivant en FOAD n'avaient-ils d'autres choix que de le faire, ou au contraire, ont-ils fait valoir et manifesté leur libre engagement par ce choix ? Que peut-on dire également des nouveaux modes de relation sociale que l'apprenant FOAD est conduit à développer, dans un dispositif qui le met à distance des enseignants et de ses pairs ?

Les développements suivants vont mettre à l'étude cet individu au cœur d'un dispositif flexible de l'époque de la flexibilité.

\subsection{Un engagement en FOAD relié au projet « emploi-formation »}

Un premier objet de la recherche était de caractériser la population des auditeurs s'inscrivant en FOAD. La première démarche, classique et néanmoins obligée, de la sociologie est de regarder les caractéristiques sociodémographiques. L'hypothèse sous-jacente est d'y trouver des déterminants qui construisent de façon spécifique l'engagement en formation. Il se révèle plutôt que la variable qui différentie les deux populations (entre le présentiel et la FOAD) correspond au projet de l'auditeur relié à son projet professionnel.

\subsubsection{L'auditeur FOAD se différentie peu de l'auditeur traditionnel du Cnam}

Les auditrices ne se distinguent pas des auditeurs : l'enquête ne fait pas ressortir de propension féminine à la FOAD liée à leur charge de famille et à l'élevage des enfants (la présence d'enfants de moins de 12 ans n'a aucun effet discriminant dans notre population). Les jeunes s'inscrivent plus volontiers en FOAD (mais il faudrait annihiler le biais introduit par le fait que l'âge des enquêtés est celui du moment de 
l'enquête, or les plus âgés avaient une palette d'UV en FOAD beaucoup plus restreint à l'époque de leur inscription).

Les «Bac+4 et plus » sont légèrement plus nombreux dans les cours en FOAD. Les $\mathrm{Bac}+3$ le sont encore plus $(56 \%$ d'entre eux sont dans des parcours de formation mixte, contre une moyenne de $46 \%$ ). Et à l'inverse, les «Bac et équivalent» sont à $44 \%$ en cours du soir (contre $26 \%$ en moyenne). Mais la prudence doit être de mise avant toute généralisation: ces données doivent être rapprochées des développements de l'offre. Il semble (sans avoir de données précises) que les cycles $\mathrm{B}(\mathrm{de} \mathrm{Bac}+2$ à $\mathrm{Bac}+4)$ ont été davantage privilégiés au fur et à mesure de la mise à disposition des UV à distance.

Les catégories socioprofessionnelles, dans notre échantillon, représentaient des effectifs, pour chacune d'entre elles, trop restreints pour pouvoir en tirer des enseignements statistiques. Mais si on regarde les statuts d'emploi ( $74 \%$ en emploi, $17 \%$ en recherche d'emploi, $7 \%$ en inactivité), la seule donnée qui ressort est la plus forte représentation des demandeurs d'emploi dans les cours du soir, pourtant plus disponibles dans la journée pour la FOAD ( $31 \%$, contre $25 \%$ en moyenne) : première indication sur le poids de la relation formation-emploi.

Mais on ne sera pas étonné de retrouver l'éloignement géographique comme facteur déterminant du choix entre FOAD et présentiel. Ainsi, $51 \%$ des auditeurs qui se considèrent très éloignés se déterminent pour la FOAD seule (contre $29 \%$ en moyenne des répondants à cette question), contre seulement $18 \%$ quand ils se considèrent «proches ». Mais ces derniers sont $51 \%$ à choisir les parcours mixtes : la variable d'éloignement géographique ne suffit donc pas, à elle seule, à déterminer les choix.

\subsubsection{Le projet «emploi-formation» conditionne le choix de la modalité pédagogique}

Plusieurs questions de l'enquête permettaient de saisir les objectifs poursuivis dans la formation. Nous avons vu qu'une des caractéristiques de l'offre du Cnam était modulaire, d'où le profil de 3 objectifs de formation : (1) l'inscription à des UV sans objectif de validation de celles-ci, (2) l'inscription à des UV avec projet de les valider (3) l'objectif d'obtention d'un diplôme. Une des questions de l'entretien téléphonique voulait saisir ce projet en amont de l'inscription (en y ajoutant un item portant sur l'indétermination de l'auditeur à ce moment-là du parcours de l'auditeur).

On constate ainsi que les auditeurs construisent un parcours de formation :

- mixte, quand l'objectif est « diplômant » (57\% contre $46 \%$ en moyenne),

- en «présentiel», quand l'auditeur n'a pas d'enjeu de validation (41\% contre $25 \%$ en moyenne) ou quand il est indéterminé ( $36 \%$ ),

- en FOAD, quand il s'agit de valider quelques UV sans inscription dans un parcours diplômant (38 \% contre 29 \% en moyenne). 
Cette première approche peut être complétée par la réponse à une question qui tentait de discriminer les projets de formation quant aux usages des savoirs. Les cours du soir apparaissent là encore comme une recherche d'une simple «actualisation des connaissances » (37\%, pour une moyenne de $25 \%)$, alors que les auditeurs qui poursuivent un parcours mixte ont pour objectif une théorie «correspondant à leur expérience». Ce dernier résultat, quand il est rapproché de celui obtenu dans le croisement précédent (visée diplômante pour les «mixtes »), peut s'interpréter comme l'objectif d'obtention du diplôme venant conforter et/ou légitimer une expérience professionnelle.

Ces résultats sont à rapprocher, pour les auditeurs en emploi $(74 \%$ de la population de l'enquête), du sentiment de blocage ou de menace sur leur emploi. Nous mobilisons-là deux variables définies par l'Observatoire des Études et des Carrières du Cnam qui sont apparues significatives pour caractériser une des dimensions de la relation à l'emploi des auditeurs. Le «blocage » fait référence à la poursuite d'une promotion professionnelle et sociale qui se trouve compromise, le sentiment de «menace» étant relié à une position défensive par rapport à l'effritement de situation acquise ${ }^{14}$.

On constate que, si les auditeurs se sentant «menacés » se répartissent également suivant les modalités pédagogiques, il n'en est pas de même pour ceux qui expriment un sentiment de «blocage », qui ont une légère propension à s'inscrire en cours du soir (ils sont $44 \%$ des auditeurs en emploi à éprouver « tout à fait » ou «plutôt » ce sentiment, et représentent $51 \%$ des inscrits en cours du soir).

Au regard de ces traitements statistiques, il est permis d'énoncer les hypothèses suivantes:

- des attentes de reconversion professionnelle (lié au sentiment de «blocage»), non encore finalisées en termes d'objectifs de formation (et, probablement, de visées professionnelles) orientent les auditeurs vers les cours du soir ;

- par contre, les objectifs de formation à visée diplômante (qui peuvent s'adosser sur un projet professionnel précis) vont s'actualiser dans des parcours utilisant au mieux l'offre de formation, en jouant en particulier de la FOAD et du présentiel.

14. L'Observatoire des Etudes et Carrières du Cnam a dégagé huit types de mobiles, qu'ils regroupent en 3 catégories pour caractériser les auditeurs du Cnam : la «promotion sociale», la «gestion rationnelle» et la «gestion sous contraintes». Dans l'enquête menée, il est difficile de reconstruire cette typologie avec les données à notre disposition. 


\subsection{Un acteur « autodéterminé » jouant des contraintes et des opportunités}

Le projet de formation, et le contexte «emploi-formation» de sa genèse, semblent donc constituer l'origine à partir de laquelle s'élaborent les modalités de parcours de formation.

Mais le projet n'est pas le seul élément: il en constitue la «matrice», mais d'autres variables participent à «configurer» le parcours. L'une d'elles a été identifiée précédemment : l'éloignement par rapport au centre d'enseignement où sont dispensés les cours du soir.

Dans ces variables, la dimension pédagogique, nous l'avons vu précédemment, apparaît seconde. Ces variables apparaissent essentiellement comme un jeu de contraintes, dans lequel l'offre de formation va apparaître comme une opportunité.

\subsubsection{Des « choix contraints»}

C'est un classique de la sociologie (ou tout au moins d'une certaine tradition sociologique) d'analyser le comportement des individus non pas à partir des choix énoncés et revendiqués par ceux qui les portent, mais à partir des éléments objectifs qui lui sont extérieurs et qui construisent ses logiques d'action.

Or, il s'agit ici davantage que cela, puisque la contrainte s'énonce en même temps que le choix. Les auditeurs (adultes, rappelons-le, et en situation professionnelle pour la grande majorité d'entre eux) ont clairement conscience de ce qu'exige l'inscription dans une formation professionnelle supérieure.

L'entrelacement de la contrainte et du choix se lit à travers l'entretien suivant d'une auditrice :

$Q$-Pourquoi vous avez entrepris cette démarche, quel était son contexte?

$R$ - Le contexte c'est que j'ai un BAC Pro, de secrétaire, j'ai trouvé une place où j'ai eu une formation dans un cabinet comptable de comptabilité, mais ils se sont contentés de me former pour ce dont ils avaient besoin, je n'avais pas les tenants ni les aboutissants ce qui n'est pas trop dans ma façon de faire... Donc j'avais envie déjà de me prouver à moi que j'étais capable de faire des études et je voulais partir dans ma branche quand même puisque ce que je voulais c'est avoir mon poste en autonomie et comprendre ce que je faisais de bout en bout. Et quand j'ai cherché pour la comptabilité, il y avait le Cnam, le Cnam ou le Cnam (rire)... Donc je me suis inscrite au Cnam parce que je n'avais pas envie d'arrêter de travailler, j'aime bien mon poste et le cours du soir c'était le plus pratique..

$Q$ - Vous avez choisi le cours du soir?

$R$ - Je ne peux pas faire autrement, j'ai une vie de famille, je travaille à plein temps, je n'ai pas pu faire... enfin je n'avais pas d'autre possibilité... Je n'ai pas eu trop le choix quoi, dans mon optique à moi, c'est-à-dire d'avoir un $B A C+2$, je n'avais le droit enfin que la possibilité du Cnam, je n'avais pas la possibilité 
de m'arrêter, comme je travaille dans une pharmacie d'officine, les postes administratifs ne sont pas reconnus, d'ailleurs ils ne sont quasiment pas dans les conventions collectives, donc je ne pouvais pas prendre un congé-formation... Donc j'ai fait le choix des cours du soir...

$Q$ - Donc vous avez fait ce choix?

$R$ - Oui (rire) et pour le moment je m'y suis tenue, je trouve ça un peu long parce que ça fait quand même plusieurs années que je suis dessus...

«Je n'avais pas eu trop le choix, quoi, dans mon optique à moi », «Je n'avais pas le droit, enfin que la possibilité »... : toutes ces hésitations sont significatives de l'élaboration même des modalités du projet. Contrainte, opportunité : le fait de conduire des études au Cnam apparaît autant comme une contrainte (je n'ai pas le choix) que comme une opportunité («je me suis inscrite au Cnam parce que je n'avais pas envie d'arrêter de travailler, ...»). La réponse à la deuxième relance est significative. Elle commence par énoncer un tas de limites au choix : "je ne peux pas faire autrement (...) je n'avais pas d'autres possibilités (...) etc.» pour terminer par : «Donc j'ai fait le choix des cours du soir ».

\subsubsection{Un « acteur » stratège de sa formation}

Mais ces éléments de contexte dans lequel se déploie l'auditeur (contrainte/opportunité), et la façon dont il construit ses choix et son parcours, ne peuvent se comprendre sans faire référence à cette « autodétermination » de l'acteur qui s'engage dans la formation.

Il s'agit ici «d'acteur », au sens de M. Crozier ${ }^{15}$, développant une stratégie, pas nécessairement consciente. Mais il s'agit aussi davantage que cela: les auditeurs sont mobilisés à partir de leur propre stratégie davantage que dans une attitude « consumériste » vis-à-vis de l'institution.

Cette dimension se perçoit, d'une certaine façon a posteriori à l'issue de leur parcours, quand les auditeurs sont mis dans une situation d'évaluation de leur formation, à travers la question de leur «satisfaction». Le taux de satisfaction est extrêmement important, puisque $36 \%$ des 352 auditeurs interviewés se disent « très satisfaits » et $53 \%$ «plutôt satisfaits » de la formation qu'ils ont suivie au Cnam. Mais sur quoi porte leur jugement ? Quels sont les critères mobilisés pour exprimer leur satisfaction de leur «formation au Cnam »? De ce point de vue, les réponses données à la question ouverte qui suivait cette question, pour leur demander d'expliciter leur réponse, est significative. Dans les raisons évoquées par les auditeurs qui se révèlent «très satisfaits » de leur formation, un nombre important (environ $50 \%$ ) exerce leur jugement à partir de critères personnels, portant essentiellement sur l'atteinte de leurs objectifs (jugement intrinsèque), $50 \%$ s'exprimant d'un point de vue extrinsèque sur la qualité de l'offre. Et ces derniers

15. Crozier, M. \& E. Friedberg (1977). L'acteur et le système. Les contraintes de l'action collective. Paris, Seuil. 
auditeurs le font souvent pour signaler une adéquation entre l'offre de formation et leur propre projet. Ce qui apparaît en jeu, donc, dans l'appréciation de leur formation pour plus de la moitié des auditeurs, est davantage eux-mêmes et leur propre démarche de formation que l'institution elle-même.

Ce résultat se confirme par le traitement d'une autre question : l'auditeur, après avoir fait le tour avec l'intervieweur sur l'usage des différentes ressources de formation, est questionné sur leur degré d'importance respectif pour sa formation. 17 des 38 auditeurs, soit près de $50 \%$, ressentent le besoin de signaler que la ressource la plus importante était eux-mêmes.

On retrouve le même résultat quand, à l'inverse, pour 37 auditeurs qui expriment leur insatisfaction de leur formation au Cnam, 7 d'entre eux renvoient la responsabilité à eux-mêmes. Ces deux auditeurs en témoignent à travers des réponses aux questions ouvertes de l'entretien téléphonique :

(1) Je n'ai pas pu répondre à la charge de travail qu'on me demandait. Ce n'est pas la faute du Cnam c'est moi-même qui ne suivais pas le rythme de travail du planning.

(2) C'est plutôt par rapport à moi car j'ai arrêté les cours.

Dans une question précise sur les raisons pour lesquelles des auditeurs ont abandonné le suivi d'une UV en FOAD, le même phénomène est observé. Ainsi, les 20 auditeurs ayant abandonné rapidement leur formation évoquent des raisons liées à la modification de leur projet personnel et de formation (5), à la modification des conditions de leur activité professionnelle (8), à la modification dans leur vie familiale et personnelle (3).

Par ailleurs, si $8 \%$ d'entre eux considèrent que le Cnam ne leur a pas apporté ce qu'ils attendaient, ils sont $28 \%$ à dire qu'il leur a permis d'atteindre "plus rapidement ses objectifs » et $60 \%$ à les avoir « accompagnés dans leur parcours ».

L'auditeur semble donc se situer dans une relation avec l'institution formatrice où il s'engage et assume son propre parcours, y compris ses échecs : cette dernière ne vient qu'en accompagnement d'une dynamique dont il a la maîtrise.

Ces différents éléments convergent pour attester d'un rapport «non consumériste » à la formation (qui traduirait un rapport « hétéro déterminé » qui se mettrait en attente des effets de l'offre institutionnelle) et d'une démarche de formation résultant d'une stratégie autodéterminée ${ }^{16}$.

16. L'attitude «consumériste » vis-à-vis de la formation est avancée par Chantal d'Halluin, pour tenter d'expliquer le peu d'engagements d'apprenants adultes dans des dispositifs d'apprentissage coopératif. Les adultes dont elle fait état seraient victimes de l'imprégnation d'une « culture de la consommation », l'obtention du diplôme étant la seule contrepartie visée de leur investissement en formation (D'Halluin C., 2001). Nos observations ne nous permettent pas un tel constat. Il nous est, bien sûr, arrivé d'interroger des auditeurs qui 
Dans cette offre flexible de l'époque de la flexibilité, les individus assument la responsabilité de leur choix. Au lieu d'une attente de prise en charge par l'institution à laquelle ils renverraient la responsabilité de leur formation, ils prennent sur eux-mêmes la responsabilité de leur engagement et les conséquences de leur choix ${ }^{17}$.

\subsection{Un acteur ambivalent}

Et pourtant là se situe une forte ambivalence : certes, les auditeurs s'engagent en assumant la responsabilité de leurs choix, mais ils en mesurent tout à la fois le coût et ses avantages.

La FOAD impose à l'auditeur d'avoir à «s'organiser» dans différentes dimensions : organisation temporelle (insérer l'acte d'apprentissage dans les temps professionnels, familiaux, etc.), organisation de l'usage des supports pédagogiques (ceux proposés, mais également les ressources complémentaires à l'initiative de l'auditeur), application d'une méthode personnelle de travail, etc.

Il faut fouiller, il faut aller par soi-même... Il n'y a pas quelqu'un qui est là derrière nous pour nous dire "ben voilà faut faire ton exercice, faut le rendre mardi... ». Non, non c'est vraiment à nous de se prendre en charge. On sait que derrière, il y a une aide, un support, un interlocuteur, mais on ne peut pas le solliciter en permanence, on ne peut pas compter sur lui quoi... Il faut vraiment se prendre en charge te grignoter des petites informations soit de par ses contacts qu'on a avec ses interlocuteurs à Laval, qu'on a eu la chance de rencontrer pendant les UV ou sur le forum de Plei@d... Il faut vraiment aller chercher les informations...

Cette dimension a été explorée par l'enquête quantitative.

\subsubsection{Une auto-organisation appréciée... et représentant un coût}

A posteriori, les auditeurs expriment le sentiment d'avoir vécu positivement cette nouvelle donne de la FOAD consistant au fait d'avoir s'auto-organiser.

A une question sur la façon dont ils «ont vécu» cette auto-organisation, ils répondent de la façon suivante : seulement $13 \%$ jugent leur expérience «plutôt négative », mais $59 \%$ la considèrent « plutôt positive » et $26 \%$ « très positive ».

considéraient leurs cours du soir au Cnam au même titre que leurs cours de danse et leurs cours de gymnastique, dans le même type de rapport que celui qui se développe dans une société de consommation de loisirs. Mais ces observations sont minoritaires. Les enjeux professionnels sont la plupart de temps en arrière-fonds de leur engagement en formation.

17. Est-il nécessaire de souligner que ce résultat ne vaut que pour les auditeurs du Cnam et n'a aucune valeur de généralisation sociologique à l'ensemble de la population. Ces derniers se soulignent par le fait de s'engager, dans un contexte de flexibilité croissant. 
Ce constat se trouve confirmée dans les réponses à une question sur les avantages de la FOAD : 85 auditeurs sur les 260 formulent cet avantage «en plein », à partir des propositions faisant référence à l'autonomie, l'organisation par soi-même, la liberté, par rapport à des avantages « en creux » ou par défaut (du type «ne pas se déplacer », réponse aux contraintes professionnelles, familiales, etc.). Il est donc possible de penser que ces auditeurs sont ceux qui apprécient le plus cette dimension de l'auto-organisation.

Or, la confrontation de leurs réponses à celle qui porte sur les inconvénients laisse apparaître que leur appréciation n'est pas sans l'expression d'une contrepartie : l'auto-organisation représente un coût. Sur ces 85 auditeurs, ils sont quelques-uns (environ 5) à s'exprimer en n'évoquant aucun inconvénient ou en les relativisant. Par exemple :

Si les supports sont mauvais c'est un désastre, sinon je ne vois que des avantages.

Mais la plupart d'entre eux évoquent essentiellement la solitude, le manque de relations avec les autres membres du groupe, la relation pédagogique difficile avec le tuteur, et très souvent le fait d'avoir à se motiver. L'extrait des réponses suivant (14 des 85) est en lui-même significatif : chaque ligne identifie un auditeur, sa réponse à la question sur les avantages, et celle à la question sur les inconvénients.

\begin{tabular}{|c|c|c|}
\hline $\begin{array}{l}\mathrm{N}^{\circ} \text { de } \\
\text { l'auditeur }\end{array}$ & AVANTAGES & INCONVÉNIENTS \\
\hline 19 & L'organisation est personnelle. & $\begin{array}{l}\text { Il faut être motivé car on n'a } \\
\text { personne derrière nous, on n'a } \\
\text { pas d'obligation. }\end{array}$ \\
\hline 38 & On s'organise comme on veut & $\begin{array}{l}\text { Il manque quelqu'un pour vous } \\
\text { motiver }\end{array}$ \\
\hline 81 & $\begin{array}{l}\text { Je trouve qu'on peut travailler comme } \\
\text { on veut et il y a moins de contraintes }\end{array}$ & $\begin{array}{l}\text { Je trouve ça plus dur de se } \\
\text { motiver car on est isolé }\end{array}$ \\
\hline 105 & Autonomie, liberté & Absence d'échange \\
\hline 108 & $\begin{array}{l}\text { Je trouve que l'autonomie est } \\
\text { importante }\end{array}$ & $\begin{array}{l}\text { Trop isolée quand on a besoin } \\
\text { d'aide }\end{array}$ \\
\hline 119 & $\begin{array}{l}\text { Gérer son apprentissage comme on le } \\
\text { souhaite, acquérir des compétences } \\
\text { d'autogestion, augmente la curiosité }\end{array}$ & $\begin{array}{l}\text { Dynamique de groupe, réponse } \\
\text { des tuteurs qui se font attendre, } \\
\text { investissement personnel difficile } \\
\text { à gérer }\end{array}$ \\
\hline 133 & $\begin{array}{l}\text { L'indépendance, rythme } \\
\text { d'apprentissage, s'organiser }\end{array}$ & Surtout d'être seul. \\
\hline 139 & La liberté dans l'organisation du travail & $\begin{array}{l}\text { C'est une perte rapide de repère } \\
\text { et cela demande beaucoup de } \\
\text { rigueur }\end{array}$ \\
\hline
\end{tabular}




\begin{tabular}{|c|c|c|}
\hline 173 & S'organiser comme on souhaite. & $\begin{array}{l}\text { Cela peut être plus difficile car } \\
\text { on est livré à soi-même. }\end{array}$ \\
\hline 174 & $\begin{array}{l}\text { Ça permet une souplesse pour le suivi, } \\
\text { c'est pratique. Ça donne une certaine } \\
\text { autonomie. L'organisation personnelle } \\
\text { est plus facile. }\end{array}$ & $\begin{array}{l}\text { La solitude ! Terrible ! C'est via } \\
\text { les échanges directs qu'on } \\
\text { apprend, à au moins } 50 \% \text {, } \\
\text { notamment pour la } \\
\text { communication, les ressources } \\
\text { humaines. L'autonomie est } \\
\text { parfois trop large et peut faire } \\
\text { perdre beaucoup de temps. }\end{array}$ \\
\hline 243 & La liberté/la souplesse & trop de souplesse justement \\
\hline 245 & Je trouve qu'on est autonome & $\begin{array}{l}\text { Je trouve qu'il manque la } \\
\text { dynamique de groupe }\end{array}$ \\
\hline 265 & $\begin{array}{l}\text { On peut s'organiser de manière } \\
\text { autonome }\end{array}$ & $\begin{array}{l}\text { On a un manque de relation avec } \\
\text { les autres élèves }\end{array}$ \\
\hline 279 & $\begin{array}{l}\text { On est très autonome, on travaille } \\
\text { quand on veut }\end{array}$ & Il faut trouver de la motivation \\
\hline
\end{tabular}

Expression de l'avantage et de l'inconvénient de la FOAD, par des auditeurs exprimant l'avantage de la FOAD en termes d'auto-organisation

Loin d'une vision enchantée de l'autonomie, il apparait donc très clairement que les auditeurs qui la recherchent et l'apprécient sont les mêmes qui par ailleurs en mesurent le coût.

4.3.2. L'attente d'un cadre socialisateur pour les auditeurs «bloqués» dans leur évolution professionnelle

Ce coût et cette capacité d'affronter l'épreuve, est-il le même selon les caractéristiques des auditeurs? De ce point de vue, les entretiens qualitatifs faisaient ressortir des différences fortes.

Deux hypothèses ont ici été privilégiées. La première est le niveau de formation initiale : on constate que le vécu de l'auto-organisation est d'autant plus positif que le niveau de formation initiale est élevé.

La deuxième hypothèse consiste à explorer la relation à l'emploi : un individu fragilisé par une précarité dans l'emploi peut être mis en difficulté dans un dispositif de formation (la FOAD) qui lui offre un espace d'échange social limité (17\% des auditeurs ont répondu être en « recherche «d'emploi »). Le témoignage de l'auditeur ci-dessous le confirme :

Je trouve que c'est exigeant. Ça crée une solitude vis-à-vis de la formation. Si vous êtes en péril professionnel ou personnel, vous ne vous en tirez pas. 
Si on exploite la distinction, définie plus haut, des auditeurs en emploi selon qu'ils expriment un «sentiment de menace » sur leur emploi ou un sentiment de «blocage» dans leur évolution professionnelle, on obtient alors les résultats suivants :

- $25 \%$ de ceux qui sont « «tout à fait » ou «plutôt » menacés » » disent avoir vécu l'auto-organisation «négativement » ou « très négativement »;

- alors que $36 \%$ de ceux qui sont «tout à fait » ou «plutôt » bloqués » » disent avoir vécu l'auto-organisation «négativement » ou « très négativement ».

Le sentiment de blocage semble donc fragiliser davantage les auditeurs, quand ils sont confrontés à «l'obligation » de l'auto-organisation dans la FOAD. On peut dès lors risquer l'interprétation de ce constat: les auditeurs exprimant ce sentiment de «blocage » peuvent être en attente d'un cadre socialisateur qui leur permet, par le contact direct avec des pairs inscrits dans une activité professionnelle et avec un intervenant du milieu professionnel (comme le permettent les cours du soir), de capter des informations pour une orientation professionnelle future et de se rassurer quant à sa pertinence et à sa faisabilité.

\subsubsection{Un renforcement de la reconnaissance de soi à la hauteur de l'épreuve}

Une des dimensions du questionnaire téléphonique voulait explorer le rapport des auditeurs avec le résultat subjectif de leur processus de formation. Alors qu'ils sont $69 \%$ en moyenne à estimer que la formation leur «a donné plus confiance » en eux, ce sentiment est partagé par $79 \%$ des auditeurs ayant suivi un parcours mixte, $76 \%$ de ceux qui ont un niveau inférieur au Bac et $86 \%$ de ceux qui expriment un «sentiment de blocage » de leur évolution professionnelle.

D'une certaine façon, ces trois données convergent: elles signifient que plus l'épreuve a été forte (par la longueur de la formation dans un parcours mixte, par un rapport à l'apprentissage moins expérimenté du fait du faible niveau de formation initial, et par la carence d'un cadre socialisateur lié à la FOAD davantage ressentie par les auditeurs se sentant «bloqués » dans leur évolution professionnelle), plus les auditeurs confortent une image de soi positive. Plusieurs auditeurs, comme le suivant, en témoignent :

D'ailleurs intérieurement j'en suis assez fier d'avoir abouti, même si à l'extérieur, ça n'a rien bougé, ça apporte... (...) Non, je ne regrette pas... je me suis prouvé à moi-même... (...) çà m'a permis de me rendre compte que j'étais capable de suivre des études, et puis c'est tout.

Cet effet, en apparence paradoxal, peut trouver son explication dans les pratiques générées par la «société du risque » dont U. Beck (2001) et A. Giddens (2004) se sont faits les analystes et dont la flexibilité dans le champ de l'emploi et du travail constitue une des dimensions. Les auditeurs se saisissent des opportunités dans un 
champ de contraintes dans un souci de réalisation de soi, qui s'avère d'autant plus conséquente que l'enjeu a été fort ${ }^{18}$.

\subsection{D’une inscription sociale «donnée » à une inscription sociale «à activer »}

Cet individu, qui se saisit de l'épreuve, n'est pas pour autant désenclavé socialement. Il entretient des relations avec ses pairs, les collègues inscrits également aux cours comme avec les intervenants et enseignants. Elles sont, évidemment, de type différent selon que l'auditeur est inscrit dans les cours du soir ou en FOAD. L'analyse longitudinale de l'offre à distance du Cnam-Pays de la Loire apporte un élément complémentaire.

Au départ, donc, les antennes. Or, ces antennes étaient situées dans des bassins d'emploi et favorisaient des interconnaissances qui s'inscrivaient dans le territoire : la dynamique collective de formation pouvait s'ancrer dans un tissu de relation de connivence par la proximité locale. Cet auditeur de l'époque des antennes en témoigne :

Au début, les premiers cours de programmation... les gens, ils se regardent, hein, quand on arrive dans la salle... on demande les infos aux autres et tout çà, et puis petit à petit, il y a la dynamique de groupe, qui arrive, et çà c'est considérable, c'est énorme, je dirais. (...) Mais tout seul, c'est épouvantable, c'est dur, ou alors à moins d'avoir un QI déjà facile à apprendre. (...).

\section{$Q$ - Les 5-6, c'était qui ?}

$R$ - C'était des collègues comme moi, d'entreprises différentes, sur Sablé, en fin de compte on était 3. Je crois que c'était moi le plus vieux. (...) Des gens tout à fait différents, d'esprit différent, mais qui avait le même, la même envie, et puis l'informatique c'était bien, à l'époque on se rencontrait, on ne faisait pas que les cours du Cnam, on discutait, on s'échangeait des trucs, et... il y avait des gens qui partageaient les mêmes besoins, peut-être pas les mêmes besoins, mais les mêmes loisirs, les mêmes passions.

18. A. Giddens caractérise ainsi une des dimensions de la «transformation de l'intimité » liée à ce qu'il appelle la radicalisation de la modernité : «Un souci de réalisation de soi, qui n'est pas une simple défense narcissique contre un mode extérieur extrêmement menaçant, sur lequel les individus ont peu d'emprise, mais aussi une appropriation positive des circonstances dans lesquelles les influences mondialisées empiètent sur la vie quotidienne » (Beck, 2004, p. 131). C. Nicole-Drancourt (2004) s'appuie sur les auteurs cités (Beck et Giddens) pour résumer des conduites qui caractérisent bien celles de nos auditeurs : «Avec les paradigmes du risque, on ne joue plus contre le risque, on trouve une réponse pour "faire avec» et c'est cette réponse qui crée l'émergence de sentiments très recherchés aujourd'hui, à savoir : le sentiment de liberté, le sentiment d'exister, une reconnaissance de soi, le besoin de nouveauté, de renouvellement, de mobilité, etc.» 


\section{D\&S - 5/2007. Industrialisation, repérages}

Ces regroupements dans les antennes ont laissé place à l'isolement physique de l'auditeur et à sa connexion via Internet. Sur les 198 auditeurs qui bénéficiaient des outils Plei@d du forum et des «chats », 50 d'entre eux (soit $25 \%$ ) ne les ont pas utilisés ; mais 132 (soit les 2/3) l'ont utilisé plus de 2 fois, et 70 (soit plus d'1/3) plus de 10 fois. Ils peuvent développer une dynamique collective qui prend le relais, de façon différente, des groupes des antennes.

En fait j'arrivais à avoir plus de renseignements en étant en téléenseignement parce que grâce à ce forum, tous les auditeurs chaque fois qu'ils ont un problème, ils peuvent poser des questions et on peut se répondre entre soi, donc ça c'est très bien parce que moi j'en aidais et d'autres m'aidaient sur les choses que je ne comprenais pas, et pour quelqu'un qui a du mal à discuter avec les autres, parce que c'est vrai que les cours du soir, ici, c'est vrai que quand on a des difficultés ce n'est pas forcément facile d'aller accoster les autres auditeurs pour leur demander de l'aide. Alors que là, sur la plate-forme, on peut poser plein de questions, qu'on nous réponde ou pas ce n'est pas grave, on peut toujours essayer...

Ces «forums » construisent des lieux d'échanges de solutions aux problèmes du cours qui fonctionnent entre une logique utilitariste et une logique « don » - « contredon ${ }^{19}$. En effet, pour qu'un tel échange puisse se développer, il faut nécessairement qu'il y ait des contributeurs de solutions. Les entretiens de la phase qualitative ont permis de montrer comment ils faisaient l'objet de pratiques utilitaristes, mais aussi d'échange qui correspondent à ce qualificatif de «don »« contre-don ».

Un auditeur témoigne de l'apport de contributeurs (lui-même et d'autres auditeurs) :

$R$ - (...) l'année dernière en base de données, j'ai beaucoup contribué à l'UV, il y a des UV où je n'avais pas de problèmes, je n'ai pas beaucoup contribué... Et je ne me sens pas assez forte pour répondre à des questions... Je ne me sens pas assez...

$Q$ - Par contre vous allez voir les réponses que donnent les collègues?

$R$ - Ah oui... oui parce qu'il y a des gens qui suivent ces UV... (...) ils en connaissaient presque autant que le tuteur... Des gens comme ça c'est vrai que ça aide...

La singularité de ces échanges «don »-«contre-don» réside, ici, dans le fait de l'ignorance physique des partenaires de l'échange: la troisième obligation décrite par M. Mauss qui consiste à « rendre » (la première étant de « donner », la deuxième, de « recevoir) » ne s'adresse pas à l'auditeur qui a «donné », mais à l'ensemble du groupe constitué autour de la formation à l'unité de valeur.

19. Notion développée par M. Mauss, pour caractériser des échanges d'un autre type que l'échange économique marchand classique : le don crée une obligation de recevoir et de rendre. 
La pratique de ces «forums » et «chats » est trop récente pour formaliser de nouvelles pratiques (et elles sont difficiles à saisir par les données quantitatives). Mais, ce qui la caractérise, c'est qu'elle repose sur une initiative à prendre par l'auditeur, comme le témoigne l'extrait d'entretien ci-dessous :

$R$-En formation à distance c'est plus individualiste, on n'ose pas trop envoyer un mail, on n'ose pas trop envoyer des problèmes dans le forum de discussion, c'est plus individualiste ...

$Q$ - Pourquoi on n'ose pas trop?

$R$ - Car on n'a pas la personne en face; et moi cela me dérange un petit peu.

Quand elle a lieu, cette nouvelle dynamique collective s'est déplacée, par rapport à la période des antennes :

- de la proximité (en co-présence) à la distance (physique) ;

- de la «convivialité » (entendue comme une appartenance à un groupe qui mobilise plusieurs registres de la relation interpersonnelle et se construit dans la durée) à des échanges orientés (centrés sur la résolution commune de problèmes, ne mobilisant qu'un registre de la relation) et réversibles (l'engagement restreint permet de se dégager d'autant plus facilement de la relation) ${ }^{20}$.

Il s'agit ici, bien sûr, de caractériser des idéaux-types, l'échange convivial étant aussi recherché par le forum (si $66 \%$ des auditeurs disent d'abord y rechercher la «solution aux problèmes posés dans le cours », ils sont la moitié à dire qu'ils en attendent aussi un «échange convivial»). Mais les auditeurs sont passés de l'appartenance donnée à un groupe social (sur lequel il pouvait s'appuyer et se reposer) à une inscription qui repose à tout moment sur l'initiative de l'individu pour activer la connexion.

\section{Conclusion : la nouvelle figure de l'individu émergeant de la flexibilité}

Tout à la fois produit reflet, et en même temps vecteur du régime de flexibilité dans le domaine de l'emploi et du travail : c'est parce qu'elle semble répondre à ces caractéristiques que l'offre de formation à distance du Cnam-Pays de la Loire a pu s'ancrer et se développer. L'ouverture qu'elle procure pour ses auditeurs à une pluralité de ressources de formation leur permet de répondre aux multiples

\footnotetext{
20. On retrouve ici plusieurs caractères des relations sociales de la «société hypermoderne » décrits par François Ascher (2005). Jean-Luc Metzger (2003) évoque, de son côté, des réseaux qu'il qualifie «d'accointance», pour singulariser ces groupes qui se distinguent des formes «sociétaires» ou «communautaires», traditionnellement distinguées par la sociologie.

Cette transformation des modes d'inscription sociale correspondent à ce qu'A. Giddens (2004) évoque à travers sa notion de dé-localisation (« extraction» des relations sociales des contextes locaux d'interaction, puis leur restructuration dans des champs spatio-temporels indéfinis »).
} 
contraintes de leur localisation géographique et de leurs contraintes temporelles en individualisant leurs pratiques formatives, quitte à ce qu'elle se paye par un confort pédagogique moindre.

Mais les possibilités nouvelles que procure cette offre flexible de l'époque de la flexibilité, en permettant et sollicitant à la fois un nouveau type de relation du formant à sa propre formation et, au-delà, à la construction de son parcours professionnel, n'est pas sans effets sur la construction de l'individu. Démêlant dans les champs de contraintes les opportunités offertes par l'extension des champs des possibles que la FOAD participe à ouvrir, revendiquant la pleine responsabilité de son engagement et de l'auto-organisation de sa formation en même temps qu'il en mesure le coût (il doit prendre l'initiative de la relation à l'autre pour sortir de son isolement et puiser dans sa motivation personnelle en dehors de tout encadrement social) : l'individu exprime une ambivalence déjà relevée dans des analyses sur la flexibilité (Berrebi-Hoffmann et al., 2005).

L'engagement est élevé, d'autant plus que capitaux culturels sont moindres, que les stratégies visent des objectifs diplômants en mobilisant tous les possibles de l'offre (le mode présentiel comme le mode à distance), ou encore que la promotion sociale et professionnelle est «bloquée »: ces engagements forts, dans une société $d u$ risque où les garanties collectives et sociales s'étiolent (Castel, 1995), procurent aussi un fort sentiment de reconnaissance de soi.

Cette recherche nous donne donc à voir la production de nouvelles formes sociales de l'individu de l'époque de la flexibilité et, au-delà, de la société du risque. Mais si cette production est activée par l'individu lui-même, l'institution formatrice est loin d'en être absente. L' «ouverture » va de pair avec la proposition de cadres formatifs où l'offre de ressources pédagogiques, à la disposition de l'auditeur, prend le pas sur le cadre «socialisateur» dans lesquelles traditionnellement elles s'inscrivaient. Nous avons vu que certains auditeurs (ceux qui se sentaient «bloqués dans leur évolution professionnelle ») semblent être davantage en attente de cette fonction «socialisatrice» qui leur permettrait une identification plus facile vers d'autres horizons professionnels.

Autrement dit, l'institution se déplace d'une fonction socialisatrice à une fonction «d'accompagnement » des parcours ${ }^{21}$.

Ces deux faits (les nouvelles formes de l'individu et le déplacement de l'institution) sont à mettre en construction conjointe : l'autodétermination, à la fois choisie et nécessaire, recherchée et coûteuse; le déplacement de l'institution

21. Dans un cadre plus global, des sociologues (Bezes et al., 2005) notent ce même déplacement de l'institution, d'une fonction socialisatrice vers des fonctions qu'ils considèrent de type informationnelles : «Les institutions d'hier façonnaient plus directement les pratiques et les représentations tandis qu'elles sont aujourd'hui davantage structurées autour d'instances intermédiaires porteuses de ressources informationnelles au service de l'action, de la coordination et du contrôle ». 
formatrice qui laisse place à l'individualisation en même temps qu'elle l'organise et l'impose.

Ce phénomène qu'il nous ait ainsi permis d'observer n'est pas isolé. Il prend sens dans les nouvelles politiques, comme le DIF (Droit individuel à la formation) négocié par les partenaires sociaux (l'avenant $\mathrm{ANI}^{22}$ ) et repris par le législateur ${ }^{23}$. Là encore, il est loisible de constater comment, tout à la fois, les institutions prennent acte du phénomène d'individualisation tout en lui donnant forme en le construisant et le renforçant. Celui-ci prend sens dans les analyses d'auteurs qui observent ce phénomène dans les différentes sphères de la vie sociale, d'un point de vue critique ${ }^{24}$, ou au contraire pour faire valoir les nouvelles formes de socialisation dont cette individualisation témoigne (Kaufmann, J. C., 2001 ; De Singly, 2003) ${ }^{25}$. Il prend sens également avec les auteurs qui la resituent historiquement dans une «radicalisation de la modernité » ou une «seconde modernité » (Beck U., 2001; Giddens A., 2004), en la mettant en regard avec la gestion des incertitudes et la prédominance du risque dans nos sociétés contemporaines.

Si les deux phénomènes coexistent et se nourrissent l'un l'autre, ils interrogent aussi leur articulation : l'accompagnement de l'institution ne peut faire fi tout à fait de la dimension «socialisatrice» qu'elle exerçait précédemment. Elle reconfigure son mode d'intervention par la mise en place, en amont de la formation, des dispositifs de bilan de compétences, de VAE ou d'orientation, autant de ressources qui renforcent encore cette individualisation, en lui donnant les moyens de sa réussite par l'accompagnement. Mais le succès n'est-il pas déjà inscrit pour une part dès le départ, dans l'engagement dans ces dispositifs individualisés et les compétences sociales qu'il suppose (qui ne pourront que se renforcer dans leur pratique même), ce dont témoignent les auditeurs de notre enquête ? Restent, dans l'ombre, les «individus par défaut » évoqués par R. Castel ${ }^{26}$, également absents du champ de notre étude, ceux dont le défaut essentiel est de ne pas bénéficier de ces compétences sociales qui leur permettent d'oser l'individualisation, et pour lesquels l'institution «socialisatrice» semble d'autant plus nécessaire. Quitte à en revisiter les formes et les moyens.

22. L'accord National Interprofessionnel a été signé le 5 Décembre 2003. Il a été prolongé par un accord sur des avenants le 23 Septembre 2005.

23. Loi du 4 mai 2004.

24. Limitons nous, ici, à R. Castel (1995) qui évoque «l'individualisme négatif» ou «l'individualisme par défaut». Ou à Alain Ehrenberg (1995), qui évoque «la fatigue d'être soi».

25. Pour ne citer qu'eux : J .-P. Kauffmann (2001) et F. De Singly (2003).

26. Castel R., op. cit. 


\section{Remerciements}

Nos remerciements, tout d'abord, aux 25 auditeurs qui se sont rendus disponibles à des entretiens approfondis et aux 352 auditeurs qui ont répondu avec beaucoup d'intérêt et d'attention à ce retour réflexif sur leur usage de la FOAD et de leur formation au Cnam au cours des entretiens téléphoniques.

Nos remerciements également à mes deux collègues de cette enquête qui, avec leur approche disciplinaire et leur connaissance intime de l'offre et de leurs auditeurs, ont partagé la collecte des données mais aussi leurs analyses : J. Moreau, sociologue et A. Meyer, Directeur Délégué, tous deux membres du Centre Régional Cnam-Pays de la Loire. A. Meyer a été l'initiateur de cette offre à distance et l'a dirigée puis accompagnée depuis ses débuts. Les résultats de cette recherche doivent beaucoup à la qualité de nos échanges.

Enfin, celle-ci n'aurait pas eu lieu sans F. Thibault, Adjoint au Directeur de la Recherche au Ministère de la Jeunesse, de l'Éducation et de la Recherche, et à l'intérêt qu'elle a manifesté à cette approche sociologique de l'étude. Qu'elle en soit également remerciée.

\section{Bibliographie}

Alava S., «Pratiques d'accompagnement et enseignement ouvert dans le Supérieur», Université ouverte, formation virtuelle et apprentissage, G. Le Meur, Paris, L'harmattan, 2002, p. 367-82.

Ascher F., La société hypermoderne, La Tour d'Aigues, L'aube, 2005.

Albero B., (dir.), Autoformation et enseignement supérieur, Paris, Hermès Science, Lavoisier, 2003.

Beck U., La société du risque, Sur la voie d'une autre modernité, Alto Aubier, 2001.

Bélisle C., Berthaud C. et al., «Etudier les usages pédagogiques des TICE: pratique de recherche ou de légitimation », Éducation Permanente, 2004, p. 139-164.

Berrebi-Hoffmann, Lallement M., Nicole-Drancourt N., Sarfati F., «Les ambivalences de la flexibilité : un point de vue français », Contribution to the 4th, International Critical Management Studies Conference, University of Cambridge, 4-6 July 2005.

Bezes P., Lallement M., Lorrain D., «Introduction, Les nouveaux formats de l'institution », Sociologie du travail, $\mathrm{n}^{\circ} 47.2005$, p. 293-300.

Boyer R., La flexibilité du travail en Europe, La Découverte, Paris, 1986.

Carré P., Moisan A., Poisson D., L'autoformation, Psychopédagogie, ingénierie, sociologie. Paris, PUF, 1997.

Castel R., Les métamorphoses de la question sociale, Une chronique du salariat, Paris, Fayard, 1995. 
CEDEFOP 2003, Lifelong Learning, citizen's views, Office des publications officielles des Communautés européennes, Luxembourg.

Correia M., Pottier F., «La « formation tout au long de la vie » : progression professionnelle ou adaptation aux contraintes », Formation Emploi n ${ }^{\circ}$ 71, 2000, p. 65-81.

Correia M., Pottier F., «La place des publics dans l'évolution d'une offre de formation, Le cas du Cnam », Education Permanente, n 164, 2005-3, p. 211-231.

De Singly F., Les uns avec les autres, Quand l'individualisme crée lien, Armand Colin, 2003.

D’Halluin C. (Dir.), «Usages d'un environnement médiatisé pour l'apprentissage coopératif », Les cahiers d'études du CUEEP, n 43, Janvier 2001.

Debon C., «Les usagers et les usages de la formation à distance proposée dans les UV Maths et statistiques du premier cycle diplômant d'Économie et Gestion du Cnam », Université ouverte, formation virtuelle et apprentissage, G. Le Meur, Paris, L'harmattan: 2002, p. 431-444.

Ehrenberg A., De l'individu incertain à la fatigue d'être soi, Pluriel, Hachette, 1995.

Fusulier B., Lannoy P. (Coord.), Les techniques de la distance, Regards sociologiques sur le télétravail et la téléformation, L'harmattan, 1999.

Giddens A., Les conséquences de la modernité, L'harmattan, 2004.

Glikman V., «Apprenants et tuteurs : une approche européenne des médiations humaines », Education Permanente, $\mathrm{n}^{\circ}$ 152, 2002, p. 55-70.

Jezegou A., Formations ouvertes, Libertés de choix et autodirection de l'apprenant, Paris, L'harmattan, 2005.

Kauffmann J. C., Ego. Pour une sociologie de l'individu, Paris, Nathan, 2001.

Kessous E., Metzger J.-L., (Dir.) Le travail avec les technologies de l'information, Ed. Lavoisier, 2005.

Lahire B., L'homme pluriel, Les ressorts de l'action, Paris, Nathan, 2001.

Metzger J.-L., La formation à distance : entre dynamique des collectifs et articulation des temps sociaux, Rapport France Telecom, 2003.

Metzger J.-L., Cléach O., «Le télétravail des cadres : entre suractivité et apprentissage de nouvelles temporalités », Sociologie du travail, $n^{\circ} 46,2004$, p. 433-450.

Mœglin P. (dir.), L'industrialisation de la formation. État de la question, Documents, Actes et rapports pour l'éducation, Paris, Centre national de documentation pédagogique.

Nicole-Drancourt C., Le risque : le produit de la régression sociale ou le coût de «l'extension du champ des possibles »? - Présentation dans le cadre du séminaire LISE-GRIOT, 2004.

Perriault J., L'accès au savoir en ligne, Odile Jacob, 2002.

Suaud C., Le sujet social (apprenant) et le «non-présentiel », Université ouverte, formation virtuelle et apprentissage, G. Le Meur. Paris, L'harmattan, 2002, p. 411-418. 